\title{
Le régime alimentaire de Schilbe multitaeniatus (Pellegrin, 1913) (Siluriforme, Schilbeidae) de la rivière Rembo Bongo au Gabon (Afrique Centrale)
}

\author{
J.F. LIWOUWOU ${ }^{1 *}$, S. Ahouansou MONTCHO ${ }^{1}$, A. CHIKOU ${ }^{1}$, D. ADANDEDJAN ${ }^{1}$, \\ J.D. MBEGA ${ }^{2}$ et A.P. LALEYE ${ }^{1}$ \\ ${ }^{1}$ Laboratoire d'Hydrobiologie et d'Aquaculture, Faculté des Sciences Agronomiques, \\ Université d'Abomey-Calavi 01 BP 526 Cotonou, Bénin. \\ ${ }^{2}$ Laboratoire d'Hydrobiologie et d'Ichtyologie, Institut de Recherches Agronomiques et \\ Forestières, CENAREST, BP 2246 Libreville, Gabon. \\ *Auteur correspondant ; E-mail : jean_feli@yahoo.fr
}

\section{RESUME}

S. multitaeniatus est un Schilbeidae très capturé et apprécié des eaux douces du Gabon. Sa bioécologie peu étudiée a fait l'objet d'une attention particulière dans l'optique de gérer durablement, de protéger et entreprendre des essais de domestication en élevage de l'espèce. De juin 2010 à décembre 2011, une étude expérimentale de son régime alimentaire a été faite dans la rivière Rembo Bongo par l'analyse de 292 estomacs de poissons pêchés aux filets dormants de mailles 10, 15, 20, 25 30, 35, 40 et $50 \mathrm{~mm}$. Les résultats ont montré que son alimentation est variée et constituée d'insectes (Io $=36,5 \%$; $I a b=29,29 \%$ ), de poissons (Io $=24,31 \%$; Iab $=18,18 \%$ ), de débris d'animaux ( $\mathrm{Io}=12,16 \%$; Iab $=9,1 \%$ ), de crustacés (Io = 4,06\%; Iab = $5,05 \%$ ), d'arachnides (Io $=1,35 \%$; Iab $=1,01 \%$ ), de fruits ( $\mathrm{Io}=13,51 \%$; $\mathrm{Iab}=28,28 \%$ ) et de débris de végétaux (Io $=6,76 \%$; Iab $=6,06 \%$ ). Cette alimentation diffère entre jeunes-adultes $(\alpha=0)$ d'une part et entre saisons de pluies et saison sèche $(\alpha=-20)$ d'autre part. Globalement, l'espèce a un régime omnivore à tendance ichtyophage, toutefois, avec une préférence d'insectes au stade jeune et de poissons au stade adulte. (c) 2013 International Formulae Group. All rights reserved.

Mots clés : Cours d'eau, Siluriforme, Schilbe multitaeniatus, alimentation.

\section{INTRODUCTION}

S. multitaeniatus (Pellegrin, 1913) est un poisson chat de la famille des Schilbeidae largement distribué en Afrique Centrale. L'espèce est connue des bassins du N'tem au Cameroun (Kandem, 1998), du Kouilou inférieur et du N'dogo au Congo Brazaville et du système Sangha/Dja au Congo (RDC) (De Vos, 1995). Au Gabon, les individus de $S$. multitaeniatus ont souvent été capturés dans le cours principal du fleuve Ogooué, dans ses affluents (Abanga, Ngounié, Missanga,
Mbiné et Mboumi), dans les lacs et zones forestières inondées et dans la Lagune Nkomi. L'espèce est parmi la plus abondante des milieux lotiques et représente avec l'espèce Schilbe grenfelli entre $33 \%$ et $40 \%$ de la biomasse des poissons capturés (Mbega, 2004). C'est également le poisson le plus recherché par les pêcheurs du fait de sa valeur marchande importante, car vendu sur les marchés à 5000 FCFA $/ \mathrm{kg}(7,6$ euros $/ \mathrm{kg})$, soit deux fois plus chère que Oreochromis schwebischi, le poisson le plus capturé dans le 
bas Ogooué (Mbega 2004). Malgré cela, l'espèce reste peu étudiée et les données portant sur son régime alimentaire sont rares alors que celles-ci constituent des informations de base pour mettre en place un programme de protection et de gestion de l'espèce ou entreprendre des études de domestication en élevage (Lalèyè, 1995). Les quelques études disponibles sur son alimentation ont révélé que l'espèce se nourrissait préférentiellement des poissons, des fruits et des coléoptères terrestres, d'insectes et du matériel végétal composé de noix et de l'herbe (De Vos, 1995 ; Mbega, 2004). Toutefois, ces informations se sont limitées au bassin inférieur de l'Ogooué et restent fragmentaires. Subséquemment, l'espèce aurait un spectre alimentaire large, quand bien même on ignore le profil général de son alimentation selon que les individus sont jeunes ou adultes. De même, on sait très peu de la variation de l'activité trophique de l'espèce selon les tailles des individus et les saisons. Aussi, la présente étude vise-t-elle la caractérisation du régime alimentaire de $S$. multitaeniatus à travers une description qualitative et quantitative des proies contenues dans les estomacs.

\section{MATERIEL ET METHODES}

\section{Milieu d'étude}

La Rivière Rembo Bongo est située au sud-ouest du Gabon à la latitude $02^{\circ} 30^{\prime}$ 'S et à la longitude $010^{\circ} 18^{\prime} \mathrm{E}$ (Figure 1). Ses caractéristiques structurales globales ne sont pas connues alors que son réseau hydrographique est assez dense avec de nombreux cours d'eau (Mboula, Tsanga, Douengui, Ngoungou...) et lacs (Goré, Mafoumi, Kivoro, Moanda...). Elle prend sa source entre 300 et $400 \mathrm{~m}$ d'altitude au nord du parc de la Moukalaba Doudou et constitue le principal cours d'eau de la limite nord-ouest dudit parc. Cette Rivière côtière coule longitudinalement sur $200 \mathrm{~km}$ vers le sud et rencontre la Lagune Ndougou $\left(434 \mathrm{~km}^{2}\right)$, la deuxième Lagune du Gabon en superficie après la Lagune Nkomi $\left(720 \mathrm{~km}^{2}\right)$. Elle est le tributaire le plus important de la Lagune Ndougou par son apport essentiel en eau douce. La Rivière est sous l'influence du climat tropical équatorial et du sous climat Lagunien (Rabenkogo, 2007). Ce dernier est caractérisé principalement par 2 saisons hydrologiques: une longue saison des pluies qui dure 8 mois (septembre-avril) et une saison sèche qui dure 4 mois (mai-aout) au cours de laquelle il persiste une vaste couverture nuageuse. La pluviométrie moyenne variant de 1900 à 2200 mm est décroissante du sud vers le nord. Le taux d'humidité élevé est de l'ordre de $86 \%$ tandis que la température varie de $24{ }^{\circ} \mathrm{C}$ à $32{ }^{\circ} \mathrm{C}$ pendant la saison des pluies et de $16^{\circ} \mathrm{C}$ à 23 ${ }^{\circ} \mathrm{C}$ pendant la saison sèche, avec des valeurs plus élevées entre mars et mai (Rabenkogo, 2007). Les individus de $S$. multitaeniatus ont été pêchés au niveau de deux stations de la Rivière Rembo Bongo (Figure 1). Les stations ont été choisies en fonction de leur accessibilité en toute saison. La station «Mafoumi » $\left(2^{\circ} 21^{\prime} 20^{\prime}\right.$ ' $\mathrm{S}$ et $10^{\circ} 09^{\prime} 38,7^{\prime}$ ' E) est située dans la partie amont de la rivière à son embouchure avec le Lac Mafoumi. La station «Moanda» $\left(2^{\circ} 26^{\prime} 03,1\right.$ ', $\mathrm{S}$ et $10^{\circ} 06^{\prime} 38,8^{\prime}$, E) est située en aval du village Ingoueka, dans le cours principal de la rivière.

\section{Echantillonnage et analyse des données}

Les pêches expérimentales mensuelles ont été réalisées pendant 2 jours de juin 2010 à décembre 2011 dans chaque station. Elles ont été faites à l'aide d'une batterie de filets maillants (mono filaments) de mailles de 10 , $15,20,2530,35,40$ et $50 \mathrm{~mm}$ placés en parallèle non loin des berges. Les filets ont été posés dans l'après-midi entre $17 \mathrm{~h} 30$ et $18 \mathrm{~h} 30$ puis relevés le lendemain matin entre 6 h et 7 h. Les poissons ont été identifiés sur le terrain selon Stiassny et al. (2007). La caractérisation du régime alimentaire a été faite sur un total de 292 estomacs examinés. Les poissons ont été pesés à l'aide d'une balance électrique Ohauss, à $0,01 \mathrm{~g}$, mesurés à l'aide d'un ichtyomètre et disséqués afin de prélever les estomacs. Les estomacs ont été prélevés et 
conservés dans des flacons de $60 \mathrm{ml}$ contenant $\mathrm{du}$ formol à $4 \%$ pour être analysés au laboratoire. Au laboratoire, chaque estomac a été ouvert par une incision longitudinale, vidé de son contenu par lavage à l'aide d'une pissette dans une boîte de pétri. Le bol alimentaire a été séparé de la poche stomacale et observé à la loupe binoculaire Olympus (20 $\mathrm{x}$ et $40 \mathrm{x}$ ). Les proies ingérées ont été déterminées et comptées. Le dénombrement a été réalisé selon les critères de Quiniou (1978): les fragments d'animaux ont été dénombrés par des têtes, le nombre d'yeux a été divisé par deux dans le cas des alevins des poissons, les amas de proies indistinctes ont été comptés comme des unités pour apporter plus de précision aux pourcentages numériques; les proies identifiées jusqu'à l'ordre ont été comptabilisées par estomac dont la digestion n'a pas été trop avancée, les fragments de crustacés (carapaces et yeux) et des poissons (écailles, arrêtes) ont été comptés et évalués comme des proies uniques. En ce qui concerne les autres groupes taxonomiques possibles (Annélides, némathelminthes, mollusques, insectes aquatiques, fourmis), il a été tenu compte de la partie la plus caractéristique (partie céphalothoracique). Lorsqu'il a été possible de compter les individus d'une même espèce (cas des annélides ou némathelminthes), une vingtaine d'animaux ont été pesés, et connaissant le poids total, le nombre a été déduit. Lorsque les proies ont été endommagées, le nombre d'individus a été déduit par comptage des pièces osseuses ou cuticulinaires identifiables. Les écailles de poissons ont été considérées comme un aliment à part entière. Les insectes ont été comptés sous loupe binoculaire et identifiés selon Tachet et al. (2000). Le volume moyen des proies (larves, d'insectes, poissons, fruits, etc.) a été évalué par déplacement d'eau à l'aide des éprouvettes graduées de différentes capacités $\left(50 \mathrm{~cm}^{3}, 25\right.$ $\mathrm{cm}^{3}, 15 \mathrm{~cm}^{3}$ ). Ensuite, par différence le volume du contenu stomacal a été déduit. Avant d'être plongés dans les éprouvettes, les constituants alimentaires ont été préalablement séchés sur du papier filtre.

\section{Expression des résultats}

Pour caractériser le régime alimentaire, quatre indices ont été utilisés :

- L’indice de vacuité (Iva) est le pourcentage du nombre d'estomacs vides (EV) par rapport au nombre d'estomacs examinés (EE). Il a été exprimé selon la relation Iva $(\%)=100 *(\mathrm{EV} / \mathrm{EE})$. L'Iva à différentes périodes de l'année renseigne sur le rythme saisonnier de l'activité alimentaire d'une population animale ou encore de l'intensité de l'alimentation (Hureau, 1970).

- L'indice d'occurrence (Io) renseigne sur les préférences alimentaires, mais n'apporte aucune indication sur l'importance quantitative des différentes proies. Il a été exprimé selon la relation Io $(\%)=$ $100 *(\mathrm{Ne} / \mathrm{Nto})$ avec Ne représentant le nombre d'estomacs contenant une proie donnée et Nto le nombre total d'estomacs non vides analysés (Lauzanne, 1976). Les proies sont considérées constantes lorsque Io > 50\%, accessoires lorsque $20 \%>$ Io $>50 \%$ et accidentelles lorsque Io $<20 \%$ (Bouhbou, 2002).

- L'indice d'abondance (Iab) ou indice numérique permet une semi quantification du régime alimentaire. Il a été exprimé par la relation $\operatorname{Iab}(\%)=100 *(\mathrm{Ni} / \mathrm{Nt})$ où $\mathrm{Ni}$ est le Nombre d'individus de la proie i (Nombre d'individus de la catégorie d'aliment i) et Nt, le Nombre total de tous les aliments consommés (Nombre d'individus des diverses catégories d'aliment) (Lauzanne, 1976).

- L'indice volumétrique (Iv) renseigne sur l'importance relative des différentes proies. Il a été exprimé par la relation Iv $(\%)=100 *$ $(\mathrm{Vx} / \mathrm{Vt})$ où $\mathrm{Vx}(\mathrm{ml})$ représente le volume individuel moyen de chaque catégorie en $\mathrm{ml}$ et Vt le volume total $(\mathrm{ml})$ des aliments intégrés (Hyslop, 1980).

Afin de comparer l'importance d'une catégorie de proie dans le régime alimentaire, et minimiser les biais possibles liés à l'utilisation des indices d'occurrence et volumétrique, l'indice alimentaire (IA) 
d'Hynes (1950) a été utilisé : IA $=(\%$ Io*\% Iv)/ 100. Cet indice renseigne aussi sur les préférences alimentaires. Les proies sont qualifiées d'importance secondaire lorsque IA $<10$, d'importance non négligeable lorsque 10 $<$ IA $<25$, d'essentielle lorsque $25<$ IA $<50$ et de largement dominantes lorsque IA $>50$ (Lauzane ,1976).

Les comparaisons du régime alimentaire entre les juvéniles (Ls $\leq 168 \mathrm{~mm}$ chez les mâles et Ls $\leq 198 \mathrm{~mm}$ chez les femelles) et les adultes (Ls $>168 \mathrm{~mm}$ chez les mâles et Ls > $198 \mathrm{~mm}$ chez les femelles) d'une part et entre les saisons d'autre part, ont été faites à l'aide de l'indice de Schoener (1970). Il a permis d'évaluer le degré de similarité entre les stades de maturité et les saisons et se note $\boldsymbol{\alpha}=1-0,5$ $\left(\sum_{i=1}^{n}\left[P_{x i}-P_{y i}\right]\right)$ Où p $\mathrm{xi}_{\mathrm{i}}$ proportion d'une proie i consommée par un stade de maturité (les individus d'une saison) $\mathrm{x}, \mathrm{p}_{\mathrm{yi}}$ proportion d'une proie i consommée par un stade de maturité (les individus d'une saison) y. Les régimes alimentaires sont considérés significativement similaires lorsque la valeur de $\boldsymbol{\alpha}$ est supérieure ou égale à 0,6 (Werner et Hall, 1977).

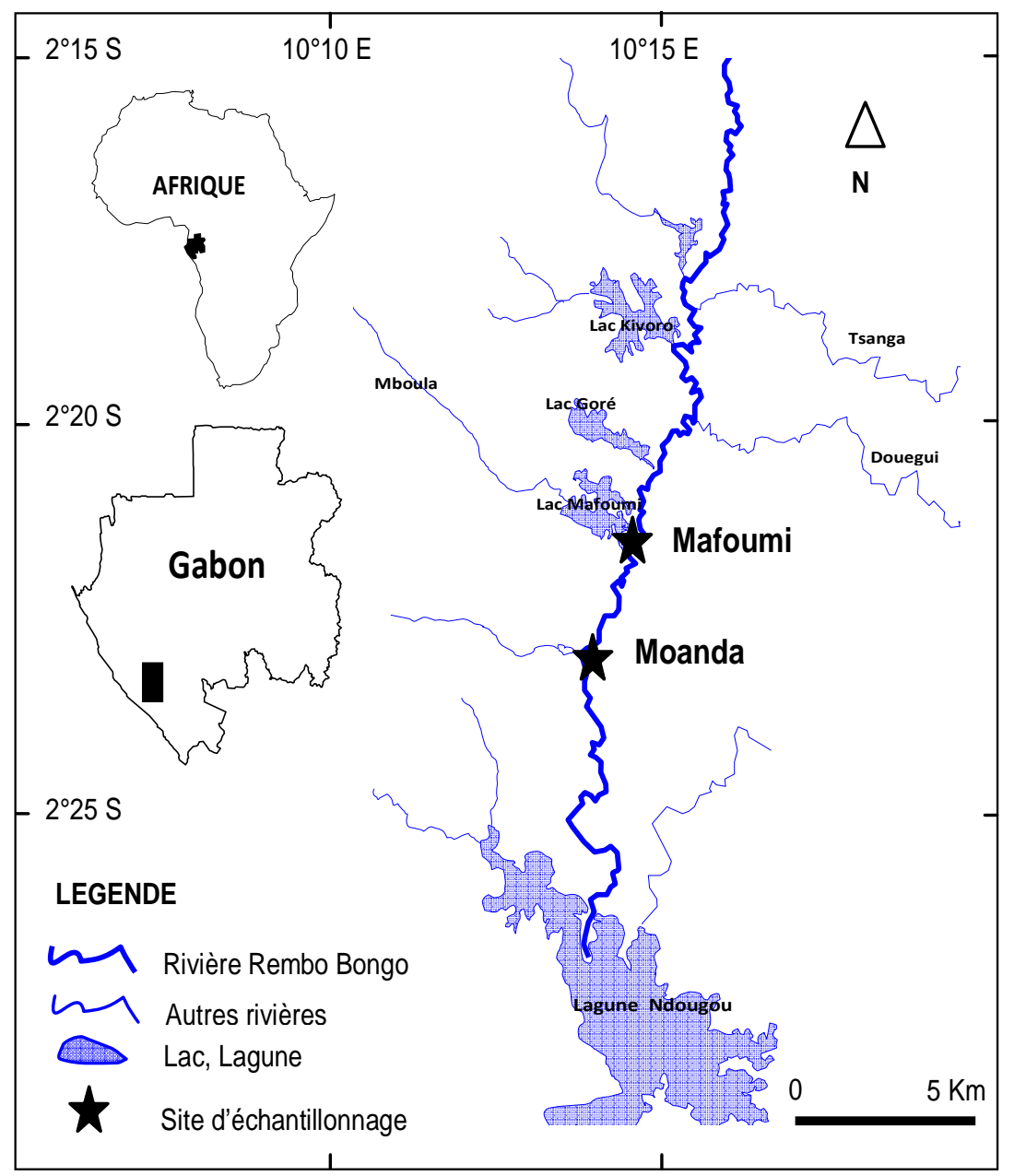

Figure 1: Rivière Rembo Bongo et stations de captures (Moanda et Mafoumi) de l'espèce Schilbe multitaeniatus au Gabon. 


\section{RESULTATS}

L'étude du régime alimentaire de S. multitaeniatus a été faite sur un total de 292 estomacs, dont 155 vides et 137 non vides. Dans les 42 estomacs appartenant aux juvéniles, 17 étaient vides et dans les 250 estomacs appartenant aux adultes, 138 étaient vides.

\section{Profil général du régime alimentaire}

Le contenu stomacal de $S$. multitaeniatus a été constitué d'une fraction animale et d'une fraction végétale, représentant un total de 17 items alimentaires (Tableau 1). La fraction animale a été composée d'insectes, d'arachnides, de crustacés et de poissons. La fraction végétale a été composée de fruits et de débris végétaux frais indéterminés. Ces débris végétaux ont été un mélange indissociable de pulpes de fruits, de graines et de petits troncs émiettés de végétaux frais (Tableau 2).

La fraction animale a été constante (Io $=79,73 \%$ ) dans le régime alimentaire et qualitativement la plus dominante $(\mathrm{Iab}=$ $65,66 \%$ ) du profil alimentaire de l'espèce. Par ailleurs, les insectes ( $\mathrm{Io}=36,5 \%$ ), les poissons (Io $=24,32 \%)$ et les fruits ( $\mathrm{Io}=13,52 \%)$ ont été les proies les plus importantes mais accessoires, tandis que tous les autres items ont été accidentels (Io < 20\%) dans le régime alimentaire (Tableaux 1 et 2).

Les insectes ( $\mathrm{Iab}=29,29 \%)$ et les fruits indéterminés ( $\mathrm{Iab}=28,28 \%$ ) ont été les items proies les plus abondants. Avec Iv $=86,47 \%$, la fraction animale a été quantitativement la plus importante du spectre alimentaire de l'espèce. Les poissons ont été les items proies les plus abondants $(\mathrm{Iv}=67,6 \%)$.

L'espèce a globalement eu une préférence pour les proies animales (IA $=$ $68,94)$. Le poisson a été une proie importante $(\mathrm{IA}=16,43)$ et les autres items proies ont été secondaires (IA < 10) dans le profil alimentaire de l'espèce.

Variation du régime alimentaire en fonction de la taille des poissons et des saisons

Les juvéniles de l'espèce ont eu une préférence pour les insectes (Io $=34 \%$ ), les fruits ( $\mathrm{Io}=18,4 \%$ ), les débris d'animaux ( $\mathrm{Io}=$ $15,8 \%$ ) et les poissons (Io $=13,15 \%$ ) (Figure 2A). Toutefois, seuls les insectes ont été accessoires $(20 \%>$ Io > 50\%) dans l'alimentation des juvéniles, tous les autres items étant accidentels (Io $<20 \%$ ). Avec respectivement Io $=10,5 \%$, les éphéméroptères et les diptères ont été les insectes les plus importants dans la composition alimentaire des juvéniles (Figure 3A1).

Les poissons (Iv $=31,8 \%)$ et les fruits ( Iv $=28,68 \%)$ ont été les items proies les plus importants chez les juvéniles (Figure 2A), tandis que les diptères ( $\mathrm{Iv}=4,2 \%)$ et les trichoptères (Iv $=3,9 \%)$ ont été par ordre d'importance les insectes les plus abondants de la composition alimentaire (Figure 3A2).

Les adultes ont eu une préférence alimentaire pour les insectes ( $\mathrm{Io}=39 \%$ ) et les poissons (Io $=36 \%$ ) (Figure $2 \mathrm{~B}$ ). Toutefois, ces deux items ont été accessoires dans l'alimentation des adultes ( $20 \%<$ Io $<50 \%$ ). Avec Io $=11 \%$, les deux insectes les plus abondants de la composition alimentaire ont été respectivement les éphéméroptères (Io = $11 \%$ ) et les odonates (11\%) (Figure 3B1). Cependant, ils ont été accidentels dans l'alimentation des adultes (Io $<20 \%$ ).

Le poisson a été le plus abondant dans les estomacs des sujets adultes ( $\mathrm{Iv}=80 \%$ ) (Figure 2B) tandis que les orthoptères (Iv = $4,7 \%)$ et les trichoptères $(\mathrm{Iv}=3 \%)$ ont été par ordre d'importance les insectes les plus consommés (Figure 3B2). 
L'indice de Schoener $(\alpha=0)$ a indiqué une différence significative entre le régime alimentaire des adultes et des juvéniles de l'espèce $S$. multitaeniatus.

En saison des pluies, les individus de l'espèce ont eu une préférence alimentaire pour 4 items proies : les insectes ( $\mathrm{Io}=44 \%$ ), les poissons (Io $=16,4 \%$ ), les débris d'animaux (Io $=13,2 \%$ ) et les fruits (Io $=$ $11 \%$ ) (Figure 4A). Cependant, seuls les insectes ont été des proies accessoires et toutes les autres des proies accidentelles. Les coléoptères (Io $=12 \%)$ ont été les insectes les plus consommés durant cette saison, quoique accidentels dans la composition alimentaire de l'espèce (Figure 5A1).

Quantitativement, le poisson (Iv = $71,3 \%)$ a été l'aliment le plus important des estomacs (Figure 4A). Les coléoptères ( $\mathrm{Iv}=$ $7,2 \%)$ ont été les insectes les plus importants en volume de proies (Figure 5A2).

En saison sèche, il y a eu la prédominance de 5 groupes d'items proies: les insectes (Io $=32,7 \%$ ), les poissons (Io $=$ $32,7 \%$ ), les débris d'animaux non identifiés (Io $=14,3 \%$ ), les fruits (Io $=8,2 \%$ ) et les débris végétaux ( $\mathrm{Io}=6,5 \%$ ) (Figure 4B). Les deux premiers ont été des items proies accessoires alors que tous les autres ont été accidentels. Les éphéméroptères (Io $=10,2 \%$ ) ont été les insectes les plus abondants dans la fraction animale (Figure 5B1).

Les insectes ( $\mathrm{Iv}=49,5 \%)$ et les poissons $(\mathrm{Iv}=23,6 \%)$ ont été relativement importants dans l'alimentation de l'espèce (Figure 4B). Les orthoptères ( $\mathrm{Iv}=21,46 \%$ ) et les trichoptères $(\mathrm{Iv}=12,34 \%)$ ont été les insectes les plus importants de la composition alimentaire (Figure 5B2).

L'indice de Schoener $(\alpha=-20)$ a indiqué une différence significative du profil alimentaire de l'espèce en saison des pluies et en saison sèche.
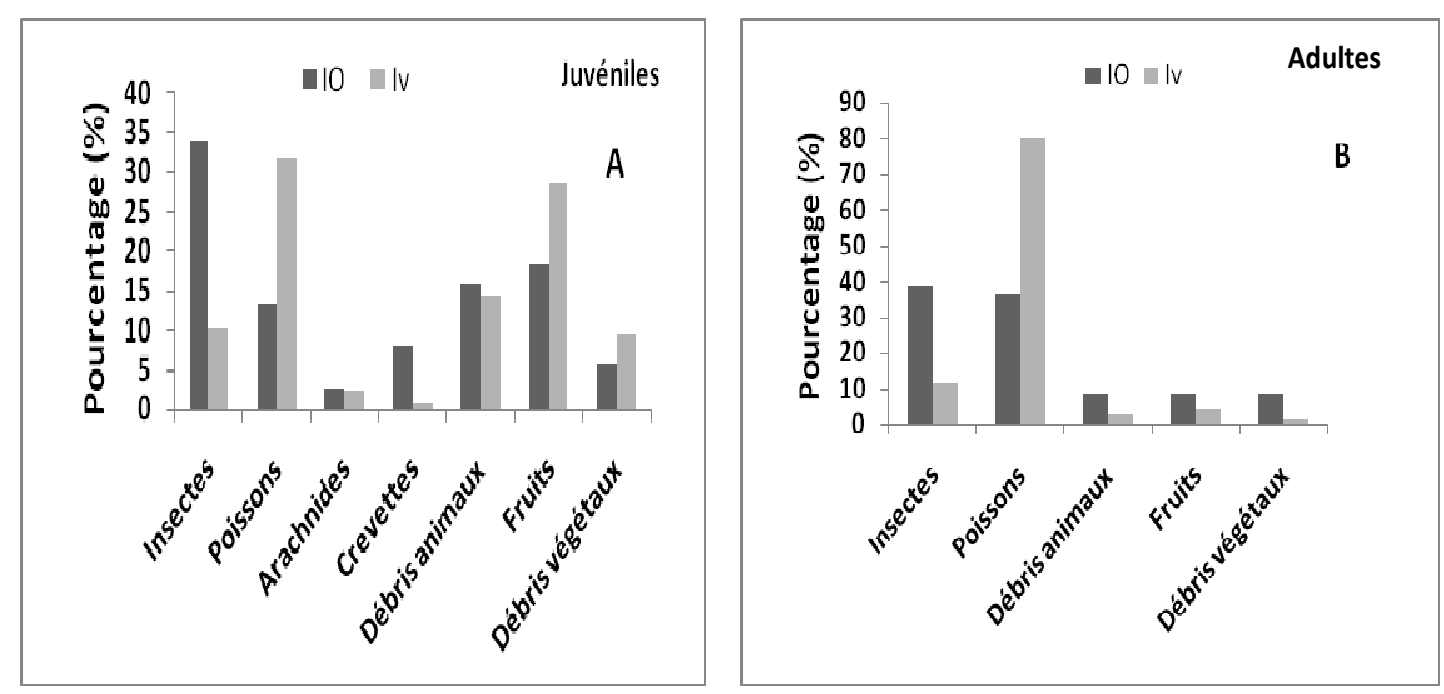

Figure 2 : Les indices d'occurrence (IO) et volumétrique (Iv) en pourcentage des grands groupes d'items proies dans les contenus stomacaux de Schilbe multitaeniatus, dans la rivière Rembo Bongo chez les juvéniles (A) et les adultes (B). 

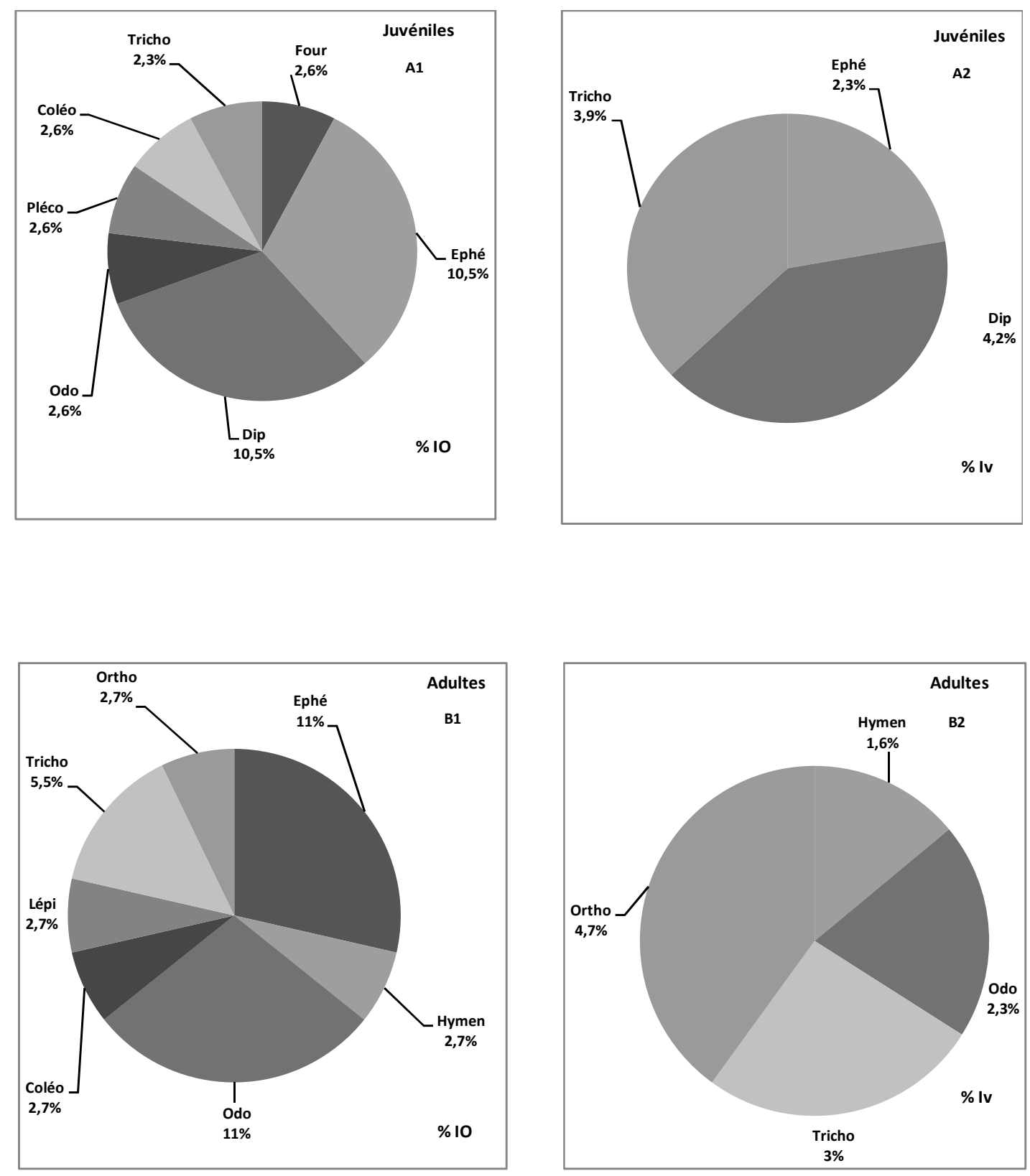

Figure 3 : Importance de chaque groupe d'insectes dans la composition alimentaire des juvéniles et des adultes de Schilbe multitaeniatus, exprimée en indices d'occurrence (A1 et B1) et volumétrique (A2 et $\mathrm{B} 2) . \% \mathrm{IO}=$ pourcentage d'indice d'occurrence $\% \mathrm{Iv}=$ pourcentage d'indice volumétrique, Coléo = Coléoptères, Pléco $=$ Plécoptères, Tricho $=$ Trichoptères, Four $=$ Fourmis, Odo $=$ Odonates, Dip $=$ Diptères, Ephé $=$ Ephéméroptères, Hymen $=$ Hyménoptères, Ortho $=$ Orthoptères, Lépi $=$ Lépidoptères . 

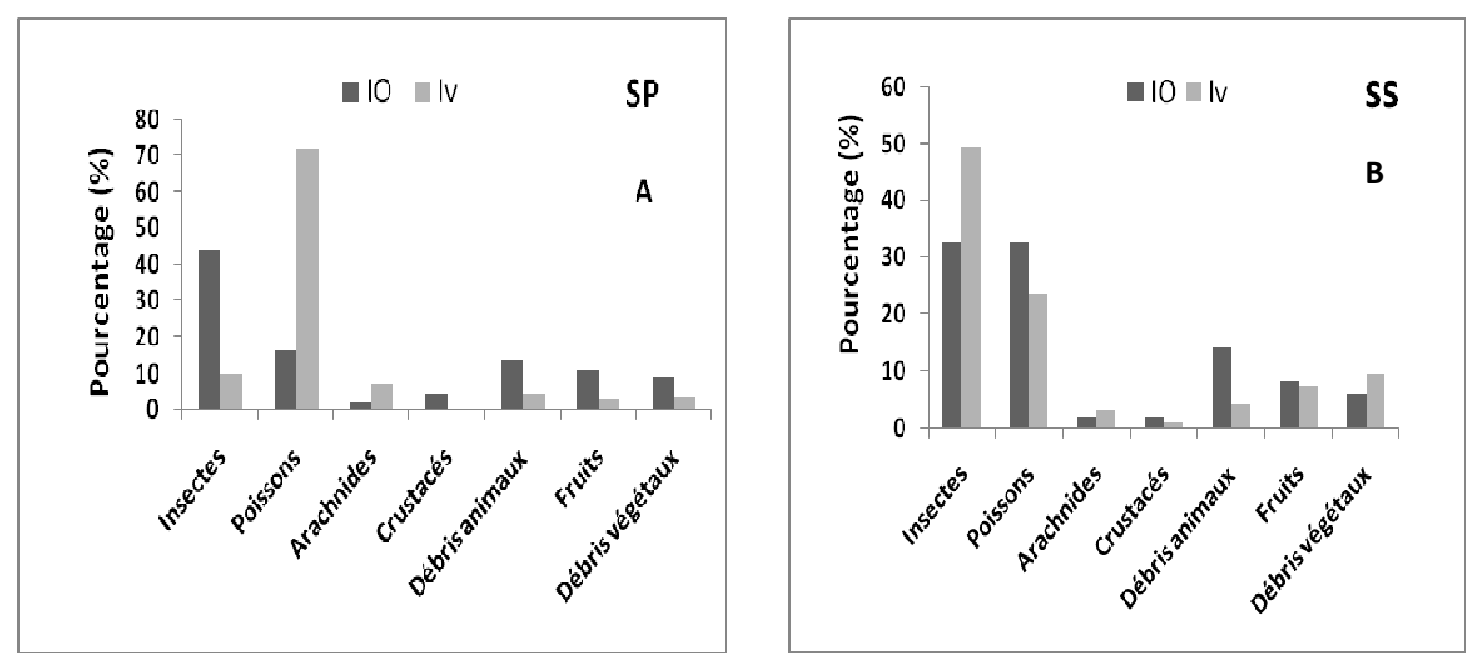

Figure 4 : Les indices d'occurrence (IO) et volumétrique (Iv) en pourcentage des grands groupes d'items proies dans les contenus stomacaux de Schilbe multitaeniatus, en saison des pluies (A) et saison sèche $(\mathrm{B})$. ( $\mathrm{SP}=$ saison des pluies ; $\mathrm{SS}=$ saison sèche).
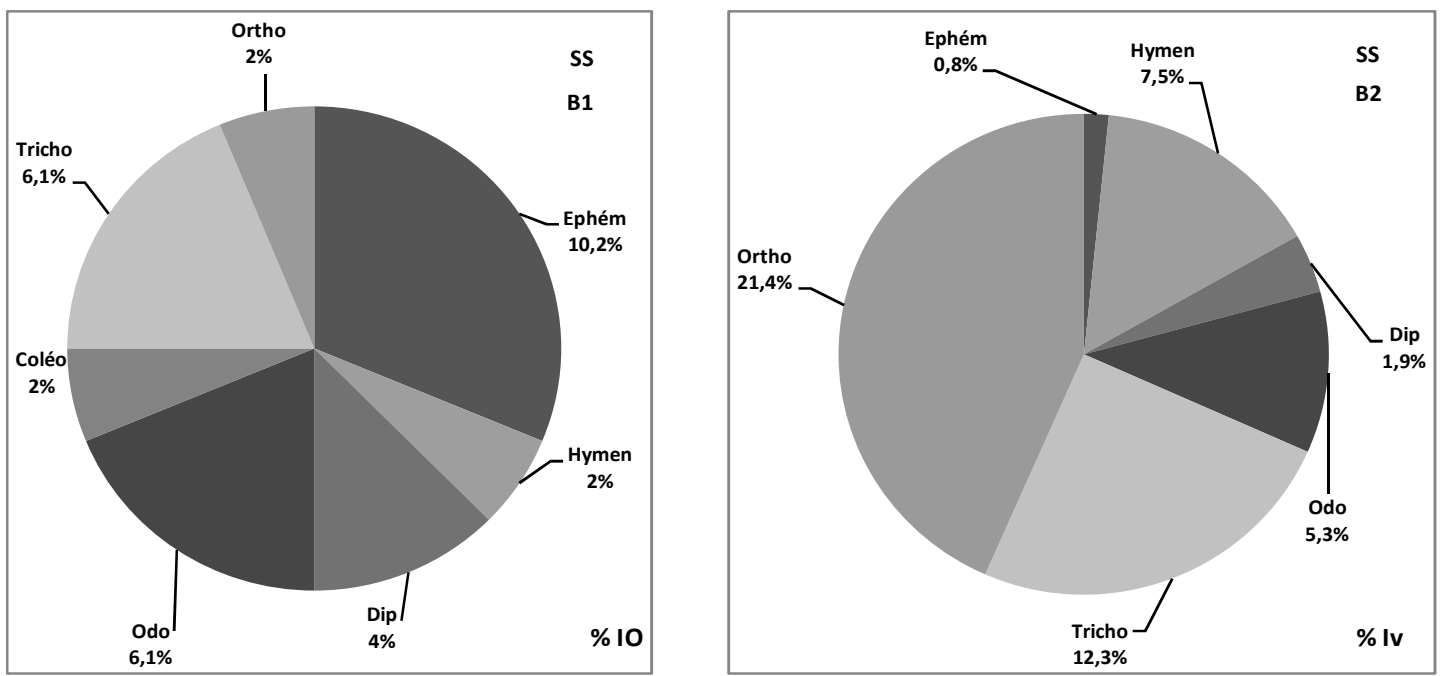

Figure 5 : Importance de chaque groupe d'insectes dans le régime alimentaire de Schilbe multitaeniatus en saison des pluies et saison sèche exprimée en indices d'occurrence (A1 et A2) et volumétrique (B1 et $\mathrm{B} 2) . \%$ IO = pourcentage d'indice d'occurrence, $\%$ Iv = pourcentage d'indice volumétrique, Coléo = Coléoptères, Pléco = Plécoptère, Tricho $=$ Trichoptères, Four $=$ Fourmis, Odo $=$ odonate, Dip $=$ Diptères, Ephé $=$ Ephéméroptères, Hymen $=$ Hyménoptères, Ortho = Orthoptères, Lépi $=$ Lépidoptères, $\mathrm{SP}=$ saison des pluie $; \mathrm{SS}=$ saison sèche . 
Tableau 1 : Composition du régime alimentaire de Schibe multitaeniatus dans la rivière Rembo Bongo.

\begin{tabular}{lccc}
\hline Items & Io \% & Iab \% & Iv \% \\
\hline Insectes & $\mathbf{3 6 , 5}$ & $\mathbf{2 9 , 2 9}$ & $\mathbf{1 2 , 0 1}$ \\
\hline Ephéméroptères & 10,81 & 9,09 & 0,57 \\
Hémiptères & 1,35 & 1,02 & 1,32 \\
Coléoptères & 2,7 & 2,01 & 0,00 \\
Odonates & 6,76 & 5,05 & 1,89 \\
Trichoptères & 4,06 & 3,03 & 3,41 \\
Orthoptères & 1,35 & 1,01 & 3,78 \\
Diptères & 5,41 & 5,05 & 1,04 \\
Fourmis & 1,35 & 1,01 & 0,00 \\
Plécoptères & 1,35 & 1,01 & 0,00 \\
Lépidoptères & 1,35 & 1,01 & 0,00 \\
Débris d'animaux & 12,16 & 9,1 & 5,58 \\
Arachnides & 1,34 & 1,01 & 0,57 \\
Poissons & 24,32 & 18,18 & 67,55 \\
Crustacés & 4,06 & 5,05 & 0,19 \\
Fruits & 14,41 & 30,77 & 10,75 \\
Débris végétaux & 7,21 & 6,66 & 3,35 \\
Total & $\mathbf{1 0 0}$ & $\mathbf{1 0 0}$ & $\mathbf{1 0 0}$ \\
\hline
\end{tabular}

$\%$ Io = indice d'occurence; \% Iab = Indice d'abondance ; \% Iv = Indice volumétrique ;

$\mathrm{IA}=$ Indice Alimentaire d'Hynes (1950).

Tableau 2: Synthèse du régime alimentaires de Schibe multitaeniatus en fractions animale et végétale dans la rivière Rembo Bongo.

\begin{tabular}{lcccc}
\hline Items & Io \% & Iab \% & Iv \% & IA \\
\hline Animaux & $\mathbf{7 8 , 3 8}$ & $\mathbf{6 2 , 6 3}$ & $\mathbf{8 5 , 9}$ & $\mathbf{6 8 , 9 4}$ \\
\hline Insectes & 36,5 & 29,29 & 12,01 & 4,38 \\
Arachnides & 1,35 & 1,01 & 0,57 & 0,008 \\
Poissons & 24,31 & 18,18 & 67,55 & 16,43 \\
Crustacés & 4,06 & 5,05 & 0,19 & 0,0095 \\
Débris d'animaux & 12,16 & 9,1 & 5,58 & 0,67 \\
\hline Végétaux & $\mathbf{2 1 , 6 2}$ & $\mathbf{3 7 , 3 7}$ & $\mathbf{1 4 , 1}$ & $\mathbf{2 , 7 4}$ \\
Fruits indéterminés & 14,41 & 30,77 & 10,75 & 1,39 \\
Débris végétaux & 7,21 & 6,6 & 3,35 & 0,217 \\
Total & $\mathbf{1 0 0}$ & $\mathbf{1 0 0}$ & $\mathbf{1 0 0}$ & \\
\hline
\end{tabular}




\section{DISCUSSION}

Durant l'étude, le spectre alimentaire de S. multitaeniatus a été large et dominé par les proies animales avec une préférence pour les poissons. Cette espèce est prédatrice avec un régime omnivore à tendance ichtyophage. Mbega (2004) avait déjà remarqué le caractère ichtyophage de cette espèce dans le bassin inférieur de l'Ogooué au Gabon. Des cas similaires ont également été signalés chez d'autres Schilbe en l'occurrence $S$. intermedius de la vallée de l'Okavango au Bostwana (Merron et Mann, 1995) et $S$. mystus de la vallée de l'Ouémé au Bénin (Chikou, 2006).

L'alimentation des juvéniles a été dans l'ensemble différente de celle des adultes ( $\alpha=$ $0)$. Au stade adulte, le régime alimentaire de l'espèce a été sélectif et dominé qualitativement et quantitativement par les poissons (Io $=36 \%$, Iv $=80 \%$ ), alors qu'au stade juvénile il a été dominé par les insectes (Io $=34 \%$, Iv $=28,68 \%$ ). Un changement de régime alimentaire avec la croissance en taille du poisson a été observé par plusieurs auteurs (Guruge 2002; Adite et al., 2005; Mbadu Zebe, 2011). Cela peut-être une adaptation pour réduire la compétition intraspécifique chez les individus de différentes classes de taille (Mbadu, 2011). Toutefois, la préférence alimentaire pour les insectes chez les juvéniles pourrait être transitoire avant d'acquérir leur régime définitif essentiellement composé de poissons à la taille adulte ; un tel constat a été respectivement fait chez $S$. mystus du bassin Tchadien (Lauzanne, 1988) et S. intermedius du Delta de l'Okavango (Merron et Mann, 1995).

Aussi, compte tenu du fait que les individus de petites et de grandes tailles aient été capturés dans le même biotope, il est possible que le changement alimentaire global observé chez l'espèce au cours de son développement soit lié à son caractère opportuniste dans le milieu ou à sa capacité de recherche de l'aliment préférentiel. En effet, l'opportunisme de ces Siluriformes a été signalé par Doumbia et al. (2008) chez $S$. mandibularis des bassins de Bia et Agnébi en Côte d'Ivoire.

Les groupes d'items proies abondants et récurrents dans la composition alimentaire de l'espèce ont été respectivement les poissons (Io $=24,31 \%$; Iab $=18,18 \%$, Iv $=$ $67,55 \%$ ), les insectes (Io $=36,5 \%$; Iab $=$ $29,29 \%$, Iv $=12,01 \%$ ), les fruits (Io $=14,41 \%$; $\mathrm{Iab}=30,77 \%, \mathrm{Iv}=10,75 \%)$ et les débris d'animaux (Io $=12,16 \%$; Iab $=9,1 \%$; Iv $=5,6 \%$ ). Toutefois, leurs importances quantitative et qualitative ont varié en fonction des saisons. Aussi, a-t-on observé que les insectes $(\mathrm{IA}=16,15)$ et les poissons $(\mathrm{IA}=7,7)$ sont les proies les plus préférées de S. multitaeniatus en saison sèche, et que les poissons (IA $=11,7)$, les insectes $(\mathrm{IA}=4,2)$, les débris végétaux $(\mathrm{IA}=3,7)$ et les fruits (IA $=3$ ) sont respectivement les aliments les plus préférés en saisons des pluies. Cette variabilité alimentaire observée en saison sèche comparativement à la saison des pluies pourrait s'expliquer par la baisse des ressources trophiques pendant cette saison. La diminution de ressources a pour corollaire la réduction de la surface du plan d'eau qui induit une baisse de la quantité d'aliments disponibles dans le milieu. Au fait, pendant les décrues, il se produit un rétrécissement de la largeur du cours d'eau pouvant parfois aller jusqu'à des points de rupture en certains endroits (discontinuité du cours d'eau) (Diomandé et al., 2009). Il s'en suit non seulement une diminution des proies disponibles, mais aussi une concentration de poissons et des insectes aquatiques sur des surfaces réduites, faisant d'eux des proies faciles pour les espèces ichtyophages ou insectivores, d'où la proportion non négligeable des insectes et des poissons dans 
l'alimentation de l'espèce durant cette période.

En saison des pluies, les fortes crues font sortir les eaux de leurs lits majeur et mineur, inondent les végétaux voisins et mettent à la disposition des poissons un nombre important d'aliments (insectes, fruits, débris végétaux...) tout en facilitant leur mobilité. En réalité sur la flore émergée, il se développe une grande quantité d'insectes terrestres divers, qui en tombant dans l'eau, constitue une nourriture facile pour les poissons insectivores. Les fourmis ( $\mathrm{Io}=3,3 \%$ ) par exemple présentes dans l'alimentation de S. multitaenitus n'ont été identifiées qu'en saison des pluies. Par ailleurs, il est connu que la plupart des plantes fructifient en cette période de l'année. Le lessivage régulier des berges des rivières dû aux pluies torrentielles, entrainent généralement vers les rivières la litière, les feuilles mortes, les insectes morts ou vivants, les fruits murs tombés, les débris végétaux divers ..., donnant ainsi à manger à différent sorte de poissons (omnivores, phytophages, insectivores,...). C'est durant cette période de l'année que le caractère éclectique et opportuniste de certaines espèces s'affirmerait le plus, les poissons utilisant les aliments disponibles et qui leur sont accessibles (Siaka Berté et al., 2009).

\section{Conclusion}

En définitive $S$. multitaeniatus de la rivière Rembo Bongo a un régime alimentaire constitué principalement d'insectes (Io = $36,5 \% ;$ Iab $=29,29 \%$, Iv $=12,1 \%)$, de poissons $(\mathrm{Io}=24,31 \%$; Iab $=18,18 \%$, Iv $=$ $67,55 \%$ ), de débris d'animaux (Io $=12,16 \%$; $\mathrm{Iab}=9,1 \%$, Iv $=5,58 \%$ ), de crustacés ( $\mathrm{Io}=$ $4,06 \%$; Iab $=5,05 \%, \quad$ Iv $=0,19 \%)$, d'arachnides ( $\mathrm{Io}=1,35 \%$; Iab $=1,01 \%$, Iv $=$ $0,57 \%$ ), de fruits (Io $=13,51 \%$; Iab $=28,28 \%$, Iv $=10,75 \%)$ et de débris végétaux (Io = $6,76 \% ; \mathrm{Iab}=6,06 \%, \mathrm{Iv}=3,35 \%)$. C'est une espèce prédatrice avec un régime omnivore à tendance ichtyophage. Cependant, on a observé dans son alimentation une préférence des insectes au stade jeune et une préférence des poissons au stade adulte. Par rapport à la saison sèche, la saison des pluies caractérisée par les hautes eaux a globalement montré une légère restriction $\mathrm{du}$ spectre alimentaire imposée par les conditions hydrolyques.

Aussi, en guise de perspectives, pour mieux cerner les moments d'alimentation chez l'espèce ou encore déterminer la vacuité chez les sujets juvéniles et adultes de l'espèce, une étude complémentaire du suivi du rythme journalier de l'activité alimentaire en 4 cycles de collecte de 24 heures s'avère nécessaire.

\section{REMERCIEMENTS}

Cet article a été réalisé dans le cadre des travaux de thèse doctorale de Jean Félicien LIWOUWOU, soutenu par le projet d'appui au programme Elargi de Formation en Gestion des Ressources Naturelles dans le Bassin du Congo et géré par le réseau des Institutions de Formation Forestière et Environnementale d'Afrique Centrale (RIFFEAC). Que ces organismes en soient remerciés. Les auteurs remercient également l'Ecole Nationale des Eaux et Forêts du Gabon, la Smithsonian Gabon, l'Agence National des Parcs Nationaux et la World Wild Fund (WWF) Gamba du Gabon pour leur soutient et appui logistique durant les deux années de collecte de données.

\section{RÉFÉRENCES}

Adite A, Winemiller KO, Fiogbé ED. 2005. Ontogenetic, seasonal, and spatial variation in the diet of Heterotis niloticus (Osteoglossiformes: Osteoglossidae) in the Sô River and Lake Hlan, Bénin, West Africa. Environnmental Biology of Fishes, 73: 367-378. 
Bouhbouh S. 2002. Bio-Ecologie de Barbus Callensis (valencienne, 1842) et $B$. Fritschi (Günther, 1874) au niveau du réservoir Allal El Fassi (Maroc). Thèse de Doctorat, Université Sidi Mohamed Abdallah, Faculté des Sciences Dhar el Mehraz Fès, Maroc, p. 126.

Chikou A. 2006. Etude de la démographie et de l'exploitation halieutique de six espèces de poissons-chats (Teleostei, Siluriformes) dans le delta de l'Ouémé au Bénin. Thèse de Doctorat, Université de Liège, Faculté des sciences, Belgique, p.469.

De Vos L. 1995. A Systematic Revision of the African Schilbeidae (Teleostei, siluriformes) (vol. 271). Annales Sciences Zoologiques. Museé Royal d'Afrique Centrale: Tervuren.

Doumbia L, Ouattara A, Gourene G. 2008. Régime Alimentaire du Poisson Chat Schilbe mandibularis (Günther, 1867) dans deux Bassins Fluviaux de Côte d'Ivoire (Bia et Agnebi). European Journal of Scientific Research, 21(2): 305-313.

Diomandé D, Doumbia L, Gouréne G. 2009. Stratégie alimentaire de deux espèces de poissons-chats dans l'hydrosystèmes fluvio-lacustre de la Bia: Synodontis bastiani Daget, 1948 et S. schall (Bloch \& Schneider, 1801). European Journal of Scientific Research, 27(1): 66-76.

Guruge WA. 2002. A food and feeding habits in three co-occuring cyprinids fishes in shallow lowland reservoir in Sri Lanka : consequences for ressource partitioning. Proceeding of the regional symposium on environment and Natural Ressources (Vol 1), Omar R, Ali Rahman Z, Latif MT, Lihan T, Adams JH (eds). Malaysia Kuala Lumpur, 10-11.

Hureau JE. 1970. Biologie comparée de quelques Poissons antarctiques
(Notothelludae). Bulletin de l'Institut Océanographique, 68: 1-244.

Hynes HBN. 1950. The food of freshwater sticklebacks (Gasterosteus aculeatus and Pygosteus pungitius) with a review of methods used in studies of the food of fishes. J. Anim. Ecol., 19: 36-58.

Hyslop EJ. 1980. Stomach contents analysis. A review of methods and their application. J. Fish Biol., 17: 411-429.

Kandem TA. 1998. Fish biodiversity of the N'tem River basin (Cameroon); Taxonomy, ecology and conservation. Thèse de Doctorat, l'Université Catholique de Louvain, Belgique, p.350.

Lalèyè P.1995. Ecologie comparée de deux espèces de Chrysichthys, poissons Siluriformes (Cloroteidae) du complexe lagunaire Lac Nokoué-Lagune de PortoNovo au Bénin. Thèse de Doctorat en sciences (zoologie), Université de Liège, Belgique, p.199.

Lauzanne L. 1988. Les habitudes alimentaires des poissons d'eau douce africains. In Biologie et Ecologie des Poissons d'Eau Douce Africains, Lévêque C, Bruton MN, Ssentongo GW (eds). ORSTOM : Paris; 221-242.

Lauzanne L. 1976. Régime alimentaire et relations trophiques des poissons du lac Tchad. Cah. Orstom, Sér. Hydrobiol., 10: $267-310$.

Mbadu Zebe. 2011. Biologie des espèces du genre Distichodus Muller et Troschel, 1845 (Distichodontidae) du pool Malebo (Fleuve Congo) en rapport avec les mécanismes d'exploitation de leurs niches trophiques. Thèse Doctorale, Faculté notre Dame de la Paix de Namur, Belgique, p.442.

Mbega JD. 2004. Biodiversité des poissons du bassin inférieur de l'Ogooué (Gabon). Thèse de Doctorat, Faculté notre Dame de la Paix de Namur, Belgique, p.390. 
Merron GS, Mann BQ. 1995. The reproductive and feeding biology of Schilbe intermedius Riippell in the Okavango Delta, Botswana. Hydrobiologia, 308: 121-129.

Quiniou. 1978. Les poissons démerseaux de la baie de Duarnenez. Alimentation et écologie. Thèse de Doctorat $3^{\mathrm{e}}$ cycle, Université de Bretagne Occidentale, France, p. 222.

Rabenkogo N. 2007. Le littoral du Nkomi (Gabon). Contribution géographique à la conservation des milieux naturels. Thèse de doctorat de géographie et Aménagement de l'espace, Université Paul Valery-Montpellier III, France, p. 307.

Schoener TW. 1970. Non-synchronous spatia overlap of lizards in patchy habitats. Ecology, 51: 408-418.
Siaka B, Essetchi KP, Ouattara NI, Tidiani K, N'douba V, Nguessan KJ. 2008. Régime alimentaire de Distichodus rostratus (Characiformes, Distichodontidae) dans un basin ouset africain (fleuve Bandama, Côte d'ivoire). Sciences \& Nature, 5(2): 167-176.

Stiassny MLJ, Teugels GG, Hopkins CD. 2007. Poissons d'Eaux Douces et Saumâtres de basse Guinée, Ouest de l'Afrique Centrale (vol. 1). IRD: Paris.

Tachet H, Richoux P, Bournaud M, Usseglio P. 2000. Invertébrés d'Eau Douce, Systématique, Biologie, Ecologie. Edition CNRS: France.

Werner EE, Hall DJ. 1977. Competition and habitat shift in two sunfishes (Centrarchidae). Ecology, 58: 869-976. 